\title{
Stereo Tracking Error Analysis by Comparison with an Electromagnetic Tracking Device
}

\author{
Matjaž Divjak and Damjan Zazula \\ System Software Laboratory, \\ Faculty of Electrical Engineering and Computer Science, \\ University of Maribor, \\ Smetanova 17, Maribor, Slovenia \\ \{matjaz.divjak, zazula\}@uni-mb.si \\ http://storm.uni-mb.si/staff.html
}

\begin{abstract}
To analyze the performance of a vision-based tracking algorithm a good reference information is needed. Magnetic trackers are often used for this purpose, but the inevitable transformation of coordinate systems can result in notable alignment errors. This paper presents an approach for estimating the accuracy of various transformation models as well as individual model parameters. Performance is evaluated numerically and then tested on real data. Results show that the method can be successfully used to analyze the tracking error of free-moving objects.
\end{abstract}

\section{Introduction}

Performance characterization of vision-based motion tracking systems is becoming an increasingly important task, especially due to a large number of existing tracking algorithms and the speed with which new ones are being presented. This issue is demonstrated by the success of the PETS Workshops [1], the PEIPA's Performance Characterization in Computer Vision project [2], as well as numerous scientific papers [3, 4]. Still, the number of papers that provide comparison with reliable ground-truth data is surprisingly low.

Authors usually manually inspect the video and mark the object positions by hand. This approach is labour intensive, unreliable and difficult to compare. To make it easier, a number of semi-automatic tools are available $[5,6]$. While such approximations to ground-truth can be very useful in certain applications, they cannot be regarded as a reliable reference because their absolute accuracy is unknown.

A more reliable approach uses a second motion tracking system with better accuracy as a reference. Magnetic tracking devices and 3D laser scanners seem the most popular choices for this purpose. Magnetic trackers feature high accuracy and speed of measurements, they are not occluded by human body and they have been in use for more than 30 years. Unfortunately, their precision is greatly affected by the presence of ferromagnetic materials and the distance from the transmitter [7, 8]. Therefore, one must take appropriate means to reduce the measurement errors before the actual experiments. On the other hand, laser scanners provide dense and incredibly accurate range measurements which can be easily used as a good reference. Main drawbacks 
are the scanning process which takes a fair amount of time (making it inappropriate for moving objects) and the operational area of contemporary devices which is quite limited. Despite that, the authors in [9] claim to be the first to provide a dense groundtruth data registered with respect to the camera.

Our tracking performance analysis is based on comparison of motion trajectories provided by the visual tracking system and the reference tracker. Each trajectory is expressed in its own coordinate system and in order to compare them, they need to be aligned accordingly. This transformation is crucial for a reliable comparison. In this paper we present a method for estimating the accuracy of various transformation approaches as well as individual model parameters. Performance of each model is numerically evaluated and then tested on real-world data. A stereocamera and a magnetic tracker are used to gather motion information.

The remainder of the paper is organized as follows: Section 2 describes the coordinate system transformation problem more formally and introduces analytical transformation models, together with a metric for comparing their performance. Section 3 starts with a comparison of numerical model properties and continues with the presentation of real-world experimental results. In Section 4 the results are compared and discussed, while Section 5 concludes the paper.

\section{Coordinate System Transformation Models}

In order to estimate the performance of a vision-based tracker by comparing it with the electromagnetic tracking device, the target's position must be measured by both systems simultaneously. First, the magnetic sensor is firmly attached to the target object. Each time an image of the scene is captured by the camera, the sensor's position is read and stored into a file, forming a motion trajectory of the object, as detected by the tracking device (a reference trajectory). Afterwards, the video is processed by a tracking algorithm to reconstruct the second trajectory. Both trajectories are expressed in their own coordinate systems (CS). In order to compare them, they need to be transformed into a common CS. Without loss of generality we selected the coordinate system of magnetic tracker $\left(\mathrm{CS}^{\mathrm{M}}\right)$ as the common one.

Obviously, the transformation itself plays a crucial role in performance characterization. If it contains errors, the two trajectories are not aligned properly and the resulting divergence does not convey the differences correctly. The following questions arise: How do we estimate which transformation is better and which is worse? Can we compare the results of two different transformations? Which parameter affects the transformation error the most?

The most frequently used method for comparing the two trajectories is aligning them by some optimization process. This approach completely ignores the possible bias errors and gives little information on how well the tracking algorithm follows the actual movement of the object. For example, if the algorithm would consistently provide exaggerated depth estimations, the "aligned" trajectories could still be a close match.

Another solution to this problem is aligning the two coordinate systems physically by carefully positioning the camera and the magnetic tracker. Although this might seem a fast and simple procedure, the alignment is never perfect and results in consid- 
erable errors in the transformation. A quick calculation shows that an orientation error of $1^{\circ}$ results in position error of $3.5 \mathrm{~cm}$ at a distance of 2 meters from the camera.

Let's assume we have a point in 3D space that needs to be expressed in both coordinate systems. In camera's coordinate system $\left(\mathrm{CS}^{\mathrm{C}}\right)$ we denote it by $\mathbf{p}^{\mathrm{C}}=\left(p_{1}^{\mathrm{C}}, p_{2}^{\mathrm{C}}, p_{3}^{\mathrm{C}}, 1\right)^{\mathrm{T}}$ and in $\mathrm{CS}^{\mathrm{M}}$ by $\mathbf{p}^{\mathrm{M}}=\left(p_{1}^{\mathrm{M}}, p_{2}^{\mathrm{M}}, p_{3}^{\mathrm{M}}, 1\right)^{\mathrm{T}}$, respectively (using homogenous coordinates). Since both vectors $\mathbf{p}^{\mathrm{M}}$ and $\mathbf{p}^{\mathrm{C}}$ represent the same point in space, we can write $\mathbf{p}^{\mathrm{M}}=\mathbf{A} \mathbf{p}^{\mathrm{C}}$, where transformation matrix $\mathbf{A}$ contains the information about translation and rotation of $\mathrm{CS}^{\mathrm{C}}$ with regards to $\mathrm{CS}^{\mathrm{M}}$. Vector $\mathbf{O}^{\mathrm{C}}=\left(o_{1}, o_{2}\right.$, $\left.o_{3}\right)^{\mathrm{T}}$ describes the position of camera's origin, while base vectors $\mathbf{i}^{\mathrm{C}}=\left(i_{1}, i_{2}, i_{3}\right)^{\mathrm{T}}, \mathbf{j}^{\mathrm{C}}=$ $\left(j_{1}, j_{2}, j_{3}\right)^{\mathrm{T}}$ and $\mathbf{k}^{\mathrm{C}}=\left(k_{1}, k_{2}, k_{3}\right)^{\mathrm{T}}$ describe its orientation. Using homogenous coordinates, matrix $\mathbf{A}$ yields the following structure:

$$
\mathbf{A}=\left[\begin{array}{cccc}
i_{1} & j_{1} & k_{1} & o_{1} \\
i_{2} & j_{2} & k_{2} & o_{2} \\
i_{3} & j_{3} & k_{3} & o_{3} \\
0 & 0 & 0 & 1
\end{array}\right] .
$$

Vectors $\mathbf{i}^{\mathrm{C}}, \mathbf{j}^{\mathrm{C}}, \mathbf{k}^{\mathrm{C}}$ and $\mathbf{O}^{\mathrm{C}}$ that define matrix $\mathbf{A}$ depend on a set of parameters $\boldsymbol{\Theta}=\left\{\Theta_{l}\right\}, l=1, \ldots, N$. Their exact number depends on the actual transformation model selected. In general, each element $a_{u, v}$ of matrix $\mathbf{A}, a_{u, v} \in \mathbf{A}$, $\forall u, v \in[1,2,3,4]$, can be described as a function of those parameters:

$$
a_{u, v}=f_{u, v}\left(\Theta_{1}, \Theta_{2}, \ldots, \Theta_{N}\right) .
$$

Of course, the camera position information is usually not available, but we could measure it by placing one of the magnetic sensors on the camera and reading its data. This approach has several shortcomings:

- The origin of $\mathrm{CS}^{\mathrm{C}}$ is usually located inside the camera body and is impossible to be measured directly.

- The camera housing is usually metallic and therefore distorts the sensor's electromagnetic field.

- While inaccurate measurements of camera position have a relatively small effect on overall accuracy, the errors in camera orientation can cause significant changes in results.

To address the abovementioned problems and to provide a way for numeric comparison we present three different models for transformation of $\mathrm{CS}^{\mathrm{C}}$ into $\mathrm{CS}^{\mathrm{M}}$. In all models the magnetic tracker is only used to measure the position of special control points which are used to calculate the orientation of the camera. For a unique solution at least three control points are needed. The following choices will be examined:

- All three control points are measured away from the camera (model A).

- Two points are measured on the camera and one away from it (model B).

- One point is measured on the camera and the other two away from it (model C). 


\subsection{Model A}

To ensure that camera body does not interfere with measurements, all three control points are measured at a certain distance from it. The camera is fixed to a flat wooden board and aligned as accurately as possible with the board's sides (Fig. 1). Three corners of the board are selected and their coordinates are measured by magnetic sensor to obtain three control points $\mathbf{T}_{1}, \mathbf{T}_{2}$ and $\mathbf{T}_{3}$. Since it is assumed that camera's coordinate axes are completely aligned with the board, the base vector $\mathbf{i}^{\mathrm{C}}$ can be expressed by $\overline{\mathbf{T}_{3} \mathbf{T}_{1}}$, the base vector $\mathbf{k}^{\mathrm{C}}$ by $\overline{\mathbf{T}_{2} \mathbf{T}_{1}}$ and the base vector $\mathbf{j}^{\mathrm{C}}$ is determined by the cross product (Fig. 1):

$$
\mathbf{i}^{\mathrm{C}}=\frac{\overline{\mathbf{T}_{3} \mathbf{T}_{1}}}{\| \overline{\mathbf{T}_{3} \mathbf{T}_{1} \|}}, \mathbf{k}^{\mathrm{C}}=\frac{\overline{\mathbf{T}_{2} \mathbf{T}_{1}}}{\left\|\overline{\mathbf{T}_{2} \mathbf{T}_{1}}\right\|}, \mathbf{j}^{\mathrm{C}}=\mathbf{k}^{\mathrm{C}} \times \mathbf{i}^{\mathrm{C}} .
$$

The position of coordinate origin $\mathbf{O}^{\mathrm{C}}$ in $\mathrm{CS}^{\mathrm{M}}$ is expressed by manually measuring relative distances $d_{1}$ and $d_{2}$ between control point $\mathbf{T}_{1}$ and $\mathbf{O}^{\mathrm{C}}$ (Fig. 1):

$$
\mathbf{O}^{\mathrm{C}}=\mathbf{T}_{1}-d_{1} \mathbf{i}^{\mathrm{C}}-d_{2} \mathbf{j}^{\mathrm{C}} .
$$

This way the transformation model A can be completely described by 11 parameters $\boldsymbol{\Theta}_{\mathrm{A}}=\left\{x_{1}, y_{1}, z_{1}, x_{2}, y_{2}, z_{2}, x_{3}, y_{3}, z_{3}, d_{1}, d_{2}\right\}, \quad$ where $\quad \mathbf{T}_{1}=\left(x_{1}, y_{1}, z_{1}\right), \quad \mathbf{T}_{2}=\left(x_{2}, y_{2}, z_{2}\right)$ and $\mathbf{T}_{3}=\left(x_{3}, y_{3}, z_{3}\right)$.

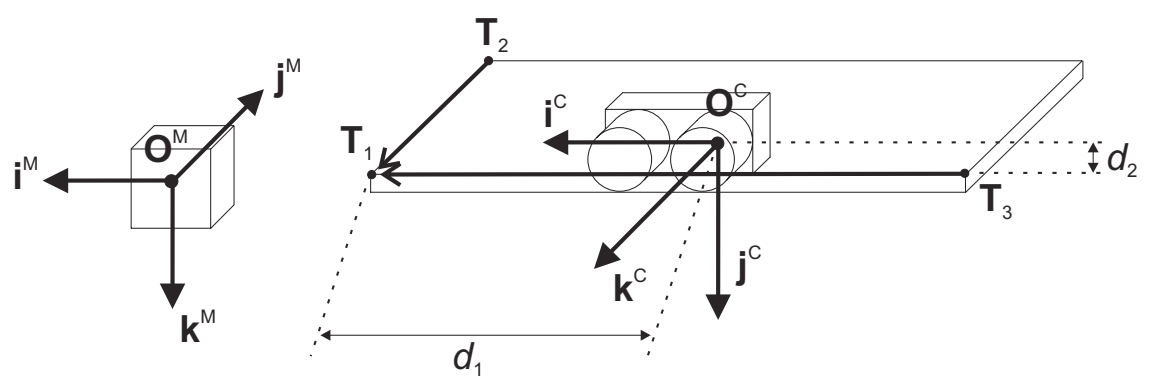

Fig. 1. Magnetic tracker (left) and the stereocamera setup (right) for model A

\subsection{Model B}

The second transformation model neglects the fact that the camera housing disturbs the measurements, because those disturbances are hoped to be very small compared to the errors caused by false orientation data. First, the position of control point $\mathbf{T}_{1}$ in front of the left camera lens (Fig. 2) is measured. Next, the magnetic sensor is attached to an arbitrary flat background (control point $\mathbf{T}_{2}$ in Fig. 2) and the camera is aligned in such a way that the sensor is visible exactly in the centre of the left stereo image. This step insures that point $\mathbf{T}_{2}$ lies on the camera's optical axis. Unfortunately, this alignment is never perfect and a mean error of $1 / 2$ pixel should be expected. As a result, $\mathbf{T}_{2}$ is displaced from the optical axis by $\Delta \mathbf{T}_{2}, \mathbf{T}_{2}^{\prime}=\mathbf{T}_{2}+\Delta \mathbf{T}_{2}$. 
Mean displacement $\Delta \mathbf{T}_{2}$ can be estimated by considering the view angle of the camera and the orientation of the background with regards to $\mathrm{CS}^{\mathrm{C}}$ (details of the derivation are omitted due to space constraints):

$$
\Delta \mathbf{T}_{2}=(r, r, r, 1)^{\mathrm{T}}, r=\frac{\left\|\mathbf{T}_{2}-\mathbf{T}_{1}\right\|}{2 \sqrt{3} f} \sqrt{\left(\frac{9.6}{v_{\mathrm{H}} \cos \alpha}\right)^{2}+\left(\frac{7.6}{v_{\mathrm{V}} \cos \beta}\right)^{2}} .
$$

Here, $f$ denotes the focal length of the lens, $v_{\mathrm{H}}$ and $v_{\mathrm{V}}$ are horizontal and vertical dimensions of camera image and $\alpha$ and $\beta$ describe the orientation of the background. Since base vector $\mathbf{k}^{\mathrm{C}}$ has the same direction as the camera's optical axis, we calculate it from $\mathbf{T}_{1}$ and $\mathbf{T}_{2}$. Similarly, base vector $\mathbf{i}^{\mathrm{C}}$ is obtained by measuring the coordinates of control point $\mathbf{T}_{3}$, positioned in front of the right camera lens (Fig. 2):

$$
\mathbf{k}^{\mathrm{C}}=\frac{\overline{\mathbf{T}_{1} \mathbf{T}_{2}^{\prime}}}{\left\|\overline{\mathbf{T}_{1} \mathbf{T}_{2}^{\prime}}\right\|}, \mathbf{i}^{\mathrm{C}}=\frac{\overline{\mathbf{T}_{\mathbf{T}}}}{\left\|\overline{\mathbf{T}_{1} \mathbf{T}_{3}}\right\|} .
$$

Base vector $\mathbf{j}^{\mathrm{C}}$ is calculated from (3), again. The origin of $C S^{\mathrm{C}}$ also lies on the camera's optical axis and is determined by displacing the point $\mathbf{T}_{1}$ by $d$ (Fig. 2):

$$
\mathbf{O}^{\mathrm{C}}=\mathbf{T}_{1}-d \mathbf{k}^{\mathrm{C}} .
$$

The transformation model $\mathrm{B}$ is therefore described by 12 parameters: $\boldsymbol{\Theta}_{\mathrm{B}}=\left\{x_{1}, y_{1}, z_{1}, x_{2}, y_{2}, z_{2}, x_{3}, y_{3}, z_{3}, d, \alpha, \beta\right\}$.

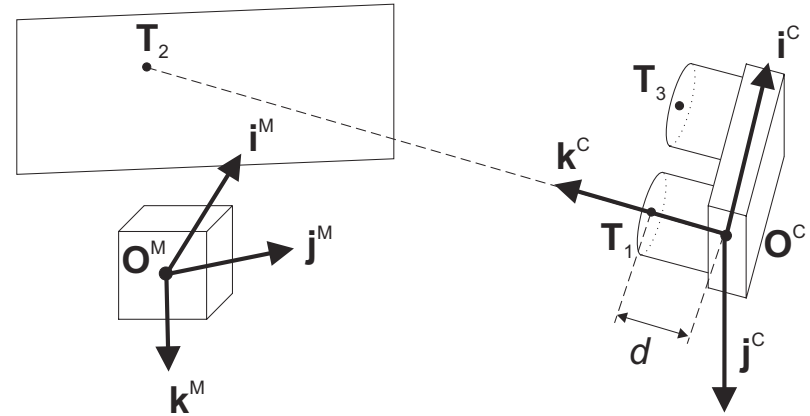

Fig. 2. Stereocamera and magnetic tracker setup for model B

The setup for model $\mathrm{C}$ is the same, except that $\mathbf{T}_{3}$ is measured anywhere on the background.

\subsection{Model C}

This model is similar to model $\mathbf{B}$, except that control point $\mathbf{T}_{3}$ is also measured on the background. The procedure for obtaining base vectors $\mathbf{k}^{\mathrm{C}}$ and $\mathbf{j}^{\mathrm{C}}$ is therefore the same. Control point $\mathbf{T}_{3}$ should be visible in the left stereo image and should also be dis- 
placed by $\Delta \mathbf{T}_{3}$ to compensate for the misorientation of the background with regards to $\mathrm{CS}^{\mathrm{C}}, \mathbf{T}_{3}^{\prime}=\mathbf{T}_{3}+\Delta \mathbf{T}_{3}$.

Vector $\overline{\mathbf{T}_{2} \mathbf{T}_{3}^{\prime}}$ is coplanar with the base vector $\mathbf{i}^{\mathrm{C}}$, but needs to be rotated around the optical axis to align it completely. The angle of rotation $\gamma=\operatorname{arctg}\left(m_{\mathrm{H}} / m_{\mathrm{V}}\right)$ is determined by horizontal $\left(m_{\mathrm{H}}\right)$ and vertical $\left(m_{\mathrm{V}}\right)$ displacement of $\mathbf{T}_{3}$ from the centre of the left stereo image. The rotated point $\mathbf{T}_{3}$ " is used to calculate the base vector $\mathbf{i}^{\mathrm{C}}$ :

$$
\mathbf{i}^{\mathrm{C}}=\frac{\overline{\mathbf{T}_{2} \mathbf{T}_{3}^{\prime \prime}}}{\| \overline{\mathbf{T}_{2} \mathbf{T}_{3}^{\prime \prime} \|}}
$$

The origin of $\mathrm{CS}^{\mathrm{C}}$ is determined by displacement $d$, as in (7), again. The third transformation model is therefore described by 14 parameters: $\boldsymbol{\Theta}_{\mathrm{C}}=\left\{x_{1}, y_{1}, z_{1}, x_{2}, y_{2}, z_{2}, x_{3}, y_{3}, z_{3}, d, \alpha, \beta, m_{\mathrm{H}}, m_{\mathrm{V}}\right\}$.

\subsection{Comparison of Models}

In order to estimate the error of transformation from $\mathrm{CS}^{\mathrm{C}}$ to $\mathrm{CS}^{\mathrm{M}}$, the sensitivity of transformation matrix $\mathbf{A}$ to the parameter set $\boldsymbol{\Theta}$ must be determined:

$$
\frac{\partial \mathbf{A}}{\partial \Theta_{l}}=\frac{\partial a_{u, v}}{\partial \Theta_{l}}=\frac{\partial f_{u, v}\left(\Theta_{1}, \Theta_{2}, \ldots, \Theta_{N}\right)}{\partial \Theta_{l}}, \text { for } \forall u, v \in[1,2,3,4], \forall l \in[1, \ldots, N] .
$$

Unfortunately, the resulting mathematical expressions are too complex for direct comparison. Instead, the derivatives are estimated numerically using parameter values from our experiments (Section 3). This gives an estimate on the largest contributor to the transformation error. In general, the mutual interaction of parameter errors is unknown, but the overall upper error bound of each model can be estimated.

The magnitude of error amplification for a certain parameter can be expressed by

$$
\begin{gathered}
\left\|\frac{\partial \mathbf{p}^{\mathrm{M}}}{\partial \Theta_{l}}\right\|=\left\|\frac{\partial \mathbf{A}}{\partial \Theta_{l}} \mathbf{p}^{\mathrm{c}}\right\| \leq\left\|\frac{\partial \mathbf{A}}{\partial \Theta_{l}}\right\| \cdot\left\|\mathbf{p}^{\mathrm{c}}\right\|, \\
S_{l}=\frac{\left\|\frac{\partial \mathbf{p}^{\mathrm{M}}}{\partial \Theta_{l}}\right\|}{\left\|\mathbf{p}^{\mathrm{M}}\right\|} \leq \frac{\left\|\frac{\partial \mathbf{A}}{\partial \Theta_{l}}\right\| \cdot\left\|\mathbf{p}^{\mathrm{c}}\right\|}{\left\|\mathbf{p}^{\mathrm{M}}\right\|}, \text { for } \forall l=1, \ldots, N .
\end{gathered}
$$

Expression (11) describes the relative sensitivity $S_{l}$ of point $\mathbf{p}^{\mathrm{M}}$ with regards to parameter $\Theta_{l}$. By inserting $\mathbf{p}^{\mathrm{C}}=\mathbf{A}^{-1} \mathbf{p}^{\mathrm{M}}$ into (11), an expression for calculating relative sensitivity of separate parameters is obtained:

$$
S_{l}=\frac{\left\|\frac{\partial \mathbf{p}^{\mathrm{M}}}{\partial \Theta_{l}}\right\|}{\left\|\mathbf{p}^{\mathrm{M}}\right\|} \leq \frac{\left\|\frac{\partial \mathbf{A}}{\partial \Theta_{l}}\right\| \cdot\left\|\mathbf{A}^{-1} \mathbf{p}^{\mathrm{M}}\right\|}{\left\|\mathbf{p}^{\mathrm{M}}\right\|} \leq \frac{\left\|\frac{\partial \mathbf{A}}{\partial \Theta_{l}}\right\| \cdot\left\|\mathbf{A}^{-1}\right\| \cdot\left\|\mathbf{p}^{\mathrm{M}}\right\|}{\left\|\mathbf{p}^{\mathrm{M}}\right\|}=\left\|\frac{\partial \mathbf{A}}{\partial \Theta_{l}}\right\| \cdot\left\|\mathbf{A}^{-1}\right\|, \text { for } \forall l=1, \ldots, N .
$$


Finally, the upper relative sensitivity limit of the whole model $\left(S^{\mathrm{MAX}}\right)$ equals the sum of separate sensitivities:

$$
S^{\mathrm{MAX}}=\frac{\left\|\frac{\partial \mathbf{p}^{\mathrm{M}}}{\partial \boldsymbol{\Theta}}\right\|}{\left\|\mathbf{p}^{\mathrm{M}}\right\|} \leq\left(\left\|\frac{\partial \mathbf{A}}{\partial \Theta_{1}}\right\|+\left\|\frac{\partial \mathbf{A}}{\partial \Theta_{2}}\right\|+\ldots+\left\|\frac{\partial \mathbf{A}}{\partial \Theta_{N}}\right\|\right) \cdot\left\|\mathbf{A}^{-1}\right\| .
$$

\section{Experiments}

Our approach was tested on real-world data using the Videre Design's STH-MD1-C [10] stereo head and Polhemus' 3Space Fastrak [11] magnetic tracker. Fastrak's static resolution is $0.8 \mathrm{~mm}$ RMS for position and $0.15^{\circ} \mathrm{RMS}$ for orientation when the sensor is within $75 \mathrm{~cm}$ from magnetic transmitter. One of Fastrak's sensors was attached to the back of the test subject's right palm. The test subject moved his palm along a predefined, physically limited path so the movement remained largely the same during all of the experiments. Three different video sequences were captured, each consisting of $120-200$ colour image pairs with $320 \times 240$ pixels.

To ensure that all transformation models were compared on the same data, the positions of all control points and other model parameters were measured before conducting the experiments. Video data was processed by our algorithm for detection of human hands and faces. 3D centroids of detected regions in each image were tracked with a predictor-corrector based algorithm [12].

Vision-based trajectories were transformed into $\mathrm{CS}^{\mathrm{M}}$ using transformation matrices of all three presented models. The parameters used to construct the matrices are shown in Table 1 and Table 2. Tables 3, 4 and 5 show the comparison results of various parameter norms. The upper sensitivity limit $\left(S^{\mathrm{MAX}}\right)$ of each model is presented in Table 6. Final performance of the presented models was estimated by calculating the RMS difference between the coordinates of the two trajectories (Table 7). In Fig. 3 an example of trajectories transformed into $\mathrm{CS}^{\mathrm{M}}$ is depicted.

Table 1. The measured parameter values for models A, B and C

\begin{tabular}{lccccccc}
\hline Parameter & $d_{1}$ & $d_{2}$ & $d$ & $\alpha$ & $\beta$ & $m_{\mathrm{H}}$ & $m_{\mathrm{V}}$ \\
Value & $490 \mathrm{~mm}$ & $14 \mathrm{~mm}$ & $48 \mathrm{~mm}$ & $10^{\circ}$ & $2^{\circ}$ & 85 pixels & 9 pixels \\
\hline
\end{tabular}

Table 2. An example of the measured coordinates of control points for models A, B and C

\begin{tabular}{lccccccccc}
\hline Parameter & $x_{1}$ & $y_{1}$ & $z_{1}$ & $x_{2}$ & $y_{2}$ & $z_{2}$ & $x_{3}$ & $y_{3}$ & $z_{3}$ \\
Model A (mm) & 201.4 & 241.9 & 91.3 & 211.2 & 522.2 & 100.2 & -68.1 & 266.0 & 124.5 \\
Model B (mm) & -83.7 & 204.8 & 88.9 & -62.4 & -99.4 & -41.7 & -97.4 & 198.8 & 84.8 \\
Model C (mm) & -83.7 & 204.8 & 88.9 & -62.4 & -99.4 & -41.7 & 107.4 & -17.7 & -10.6 \\
\hline
\end{tabular}


Table 3. Parameter norms for model A

\begin{tabular}{lcccccc}
\hline Parameter & $x_{1}$ & $y_{1}$ & $z_{1}$ & $x_{2}$ & $y_{2}$ & $z_{2}$ \\
$S_{l}$ & 398.5 & 175.2 & 174.8 & 1.4 & 0.7 & 19.9 \\
Cond. no. & 11145.6 & 7744.0 & 2716.1 & 1.9 & 9.8 & 14.0 \\
\hline Parameter & $x_{3}$ & $y_{3}$ & $z_{3}$ & $d_{1}$ & $d_{2}$ & \\
$S_{l}$ & 10.6 & 224.3 & 224.3 & 398.6 & 398.6 & \\
Cond. no. & 620.5 & 14590.5 & 490.5 & 1 & 1 & \\
\cline { 1 - 4 } & & & & & &
\end{tabular}

Table 4. Parameter norms for model B

\begin{tabular}{lcccccc}
\hline Parameter & $x_{1}$ & $y_{1}$ & $z_{1}$ & $x_{2}$ & $y_{2}$ & $z_{2}$ \\
$S_{l}$ & 410.4 & 390.7 & 410.2 & 20.4 & 3.4 & 20.3 \\
Cond. no. & 25408.7 & 4817.2 & 5326.1 & 1269.5 & 54.7 & 48.1 \\
\hline Parameter & $x_{3}$ & $y_{3}$ & $z_{3}$ & $d$ & $\alpha$ & $\beta$ \\
$S_{l}$ & 0.4 & 6.9 & 4.5 & 390.1 & 5.2 & 1.1 \\
Cond. no. & 2.3 & 4.5 & 1 & 1 & 67.4 & 67.4 \\
\hline
\end{tabular}

Table 5. Parameter norms for model C

\begin{tabular}{lccccc}
\hline Parameter & $x_{1}$ & $y_{1}$ & $z_{1}$ & $x_{2}$ & $y_{2}$ \\
$S_{l}$ & 408.8 & 389.1 & 408.5 & 20.3 & 3.4 \\
Cond. no. & 1163619. & 136461. & 134923. & 558360. & 25446.2 \\
\hline Parameter & $z_{2}$ & $x_{3}$ & $y_{3}$ & $z_{3}$ & $d$ \\
$S_{l}$ & 20.2 & 0.1 & 0.1 & 0.8 & 388.5 \\
Cond. no. & 11006.3 & 1 & 1 & 1 & 1 \\
\hline Parameter & $\alpha$ & $\beta$ & $m_{\mathrm{H}}$ & $m_{\mathrm{V}}$ & \\
$S_{l}$ & 5.2 & 1.1 & 0.5 & 4.6 & \\
Cond. no. & 771576.9 & 771576.9 & 1 & 1 & \\
\hline
\end{tabular}

Table 6. The upper sensitivity limit $\left(S^{\mathrm{MAX}}\right)$ of models A, B and C

\begin{tabular}{cccc}
\hline Model & A & B & C \\
$S^{\text {MAX }}$ & 2026.9 & 1661.1 & 1651.2 \\
\hline
\end{tabular}

Table 7. The RMS difference between the coordinates of video-based and magnetic-based trajectories. Mean values for three experiments are shown

\begin{tabular}{ccccc}
\hline Model & $\begin{array}{c}\mathbf{x} \text { RMS } \\
\text { difference } \\
(\mathbf{m m})\end{array}$ & $\begin{array}{c}\text { y RMS } \\
\text { difference } \\
(\mathbf{m m})\end{array}$ & $\begin{array}{c}\mathbf{z} \text { RMS } \\
\text { difference } \\
(\mathbf{m m})\end{array}$ & $\begin{array}{c}\text { Total RMS } \\
\text { difference } \\
(\mathbf{m m})\end{array}$ \\
\hline A & $16.1 \pm 6,1$ & $5.8 \pm 1.2$ & $10.7 \pm 1.7$ & $20.5 \pm 4.9$ \\
B & $14.8 \pm 2.9$ & $12.7 \pm 0.7$ & $72.5 \pm 6.4$ & $75.1 \pm 6.8$ \\
C & $34.7 \pm 3.2$ & $12.7 \pm 0.4$ & $55.1 \pm 6.7$ & $66.4 \pm 6.9$ \\
\hline
\end{tabular}



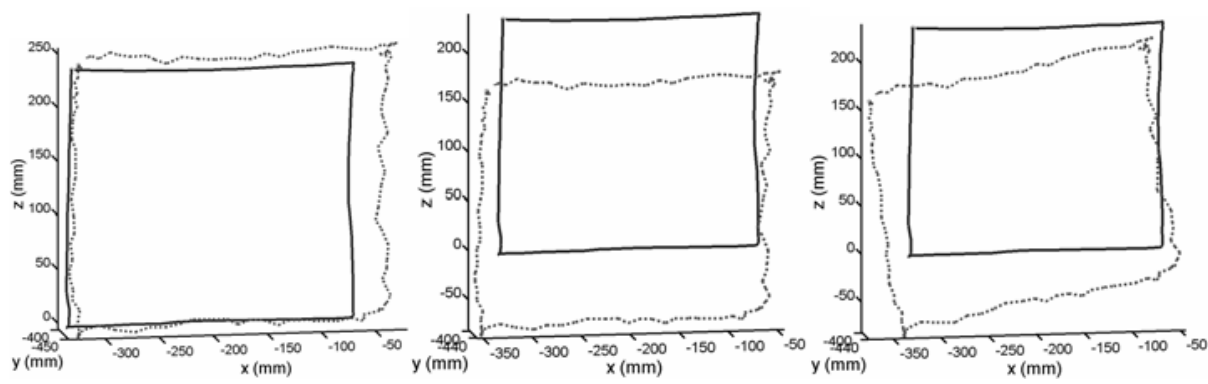

Fig. 3. Transformation of vision-based trajectories into $\mathrm{CS}^{\mathrm{M}}$. Magnetic tracker data is depicted by solid lines, the stereocamera data is depicted by dotted lines (model A, model B, model C)

\section{Discussion}

With careful selection of parameters each presented model can be shown to give the best transformation results. However, if a fixed set of parameters is given this limits the tests to one specific setup of stereocamera and Fastrak. All numerical results and conclusions are thus valid for this selected setup only. The methodology is however universally applicable.

A quick glance at Tables 3, 4 and 5 reveals which model parameters are the most sensitive and thus contribute the majority of the transformation error. For model A this is $\mathbf{T}_{1}, \mathbf{T}_{3}, d_{1}$ and $d_{2}$, with $x_{1}, d_{1}$ and $d_{2}$ being the most sensitive. For models $\mathrm{B}$ and $\mathrm{C}$ parameters $\mathbf{T}_{1}$ and $d$ are the most sensitive. Also the model $\mathrm{C}$ has a large number of badly conditioned parameters. Comparison of the estimated $S^{\mathrm{MAX}}$ values shows that models $\mathrm{B}$ and $\mathrm{C}$ have similar sensitivity, but model $\mathrm{A}$ is the most sensitive and, therefore, the most error-prone (Table 6).

However, the results of actual trajectory comparison show a different picture. Transformation with model A resulted in the smallest RMS difference of all three models, while model B is having the worst results (Table 7, Fig. 3). How is this possible?

We have to consider that if a certain parameter has large sensitivity and small actual value, its effect on the transformation can be smaller than from a parameter with small sensitivity and large actual value. The main advantage of model $\mathrm{A}$ is that all three control points are measured at a certain distance from the camera (approx. 50 $\mathrm{cm}$ in our experiments). If a certain measurement error is made, its effect on the camera orientation is by far smaller than if the same error is made only a few $\mathrm{cm}$ away from the camera. The selected parameter set clearly makes models B and C more error-prone than model A. For example, even though parameters $\alpha$ and $\beta$ have negligible sensitivity, it is clear that an orientation error of several degrees would have devastating effects on the transformation.

Fastrak's reference measurements also contain certain amount of error $(0.8 \mathrm{~mm})$, but since it is much smaller in comparison to video errors, it can be neglected. However, if the magnetic sensor is used outside the perimeter of the highest accuracy, those errors should be taken into consideration and compensated accordingly. 


\section{Conclusion}

By analyzing the worst-case sensitivity of various transformation models a limited comparison of those models is possible. The most influential parameters can easily be identified, but the actual parameter values used also have a significant effect on the final transformation error. By careful selection of parameters, any model can be shown to perform the best. Therefore, such comparisons are only reasonable if the parameters are fixed to a certain setup of camera and magnetic tracker.

In our experiments the transformation model $\mathrm{A}$ was able to align the vision-based and magnetic-based trajectories reasonably well. The remaining total coordinate difference of $20 \mathrm{~mm}$ (RMS) is believed to be caused mostly by our tracking algorithm. Further classification of this error into the errors induced by the transformation and our tracking algorithm would provide more accurate discrepancy estimates, making it possible to use the tracker as an absolute ground-truth reference. However, this task remains for the future work.

\section{References}

1. IEEE International Workshop on Performance Evaluation of Tracking and Surveillance (PETS). IEEE Winter Vision Multi-Meeting. Breckenridge, Colorado (2005) http://pets2005.visualsurveillance.org/

2. Performance Characterization in Computer Vision. PEIPA - Pilot European Image Processing Archive. http://peipa.essex.ac.uk/index.html

3. Christensen, H.I., Förstner W.: Performance characteristics of vision algorithms. Machine Vision and Applications, Vol. 9, No. 5-6. Springer-Verlag (1997), 215-218

4. Gavrila, D.M.: The visual analysis of human movement: A survey. Computer Vision and Image Understanding, Vol. 73, No. 1.Academic Press (1999) 82-98

5. Doermann, D., Mihalcik, D.: Tools and Techniques for Video Performance Evaluation. Int. Conf. on Pattern Recognition ICPR 2000. Barcelona (2000) 4167 - 4170

6. Black, J., Ellis, T., Rosin, P.: A Novel Method for Video Tracking Performance Evaluation. The Joint IEEE International Workshop on Visual Surveillance and Performance Evaluation of Tracking and Surveillance. Nice (2003) 125-132

7. La Cascia, M., Sclaroff, S., Athitsos, V.: Fast, Reliable Head Tracking under Varying Illumination: An Approach Based on Registration of Texture-Mapped 3D Models. IEEE Trans. Pattern Analysis and Machine Intel., Vol. 22, No. 4, (2000) 322-336

8. Yao, Z., Li, H.: Is A Magnetic Sensor Capable of Evaluating A Vision-Based Face Tracking System? Conf. on Computer Vision and Pattern Recognition Workshop, Vol. 5. Washington (2004)

9. Mulligan, J., Isler, V., Daniilidis, K.: Performance Evaluation of Stereo for Tele-presence. Int. Conf. on Computer Vision ICCV 2001, Vol. 2. Vancouver (2001)

10. Videre Design, STH-MD1/-C Stereo Head. User's Manual (2001)

11. Polhemus Inc., 3Space Fastrak User's Manual (1998)

12. Divjak, M.: 3D Motion Tracking Using a Stereocamera. Master Thesis. Faculty of Electrical Engineering and Computer Science, University of Maribor. Maribor (2003) 